\title{
A Proximal Characterization of the Reachable Set
}

\author{
F. H. Clarke*
}

CRM-2215

October 1994 


\begin{abstract}
We show that the graph of the reachable set of a control system given by a differential inclusion is uniquely characterized by a Hamilton-Jacobi equation involving proximal normals.
\end{abstract}

\title{
Résumé
}

On démontre que le graphe de l'ensemble des points accessibles d'un système de contrôle décrit par une inclusion différentielle est caractérisé par une équation Hamilton-Jacobi impliquant les normales proximales. 
We study a control system defined via a differential inclusion

$$
\dot{x}(t) \in F(t, x(t)) \text { a. e. }
$$

As usual, a trajectory of (1) refers to an absolutely continuous function $x(\cdot)$ satisfying (1) on a given interval $[a, b]$. The equivalence of (1) to a classical control system $\dot{x}=f(t, x, u), u \in U$ is well-understood; we shall not dwell upon it. For a given choice of initial time $t_{0}$ and nonempty compact subset $A$ of $\mathbb{R}^{n}$, we consider the set $\mathcal{R}$ defined as follows:

$$
\mathcal{R}=\{(\sqcup, \S(\sqcup)): \sqcup \geq \sqcup, \S(\cdot) \text { is a trajectory on }[\sqcup,, \sqcup], \S(\sqcup,) \in \mathcal{A}\} \text {. }
$$

The assumptions on the multifunction $F$ are as follows:

(H1) For each $(t, x) \in\left[t_{0}, \infty\right) \times \mathbb{R}^{n}$, the set $F(t, x)$ is a nonempty, convex, compact subset of $\mathbb{R}^{n}$.

(H2) For some constants $\gamma$ and $c$, and for all $(t, x)$ in $\left[t_{0}, \infty\right) \times \mathbb{R}^{n}$, one has

$$
v \in F(t, x) \Rightarrow|v| \leq \gamma|x|+c .
$$

(H3) $F$ is locally Lipschitz on $\left[t_{0}, \infty\right) \times \mathbb{R}^{n}$; i.e., for any bounded subset $S$ of $\left[t_{0}, \infty\right) \times \mathbb{R}^{n}$ there is a constant $K$ such that, for all $\left(t_{i}, x_{i}\right) \in S(i=1,2)$, we have

$$
F\left(t_{2}, x_{2}\right) \subseteq F\left(t_{1}, x_{1}\right)+K\left|\left(t_{2}-t_{1}, x_{2}-x_{1}\right)\right| \bar{B},
$$

where $B$ denotes the closed unit ball in $\mathbb{R}^{n}$.

It is a well-known fact that under these hypotheses the set $\mathcal{R}$ is closed, and that its "slice" at time $T$, the reachable set $\mathcal{R}_{\mathcal{T}}:=\{\S:(\mathcal{T}, \S) \in \mathcal{R}\}$ is compact and nonempty for each $T \geq t_{0}$.

A proximal normal [3] $\zeta$ to a closed set $S$ at a point $x \in S$ is a vector $\zeta$ such that, for some $\sigma \geq 0$, one has

$$
\left\langle\zeta, x^{\prime}-x\right\rangle \leq \sigma\left|x^{\prime}-x\right|^{2} \quad \forall x^{\prime} \in S .
$$

The set of proximal normals to $S$ at $x$ is a cone; we denote it $\partial_{P} S(x)$. Note that $0 \in \partial_{P} S(x) \quad \forall x \in S$, and that $\partial_{P} S(x)$ is undefined when $x \notin S$. It is known that $\partial_{P} S(x)$ reduces to the set of normals in the usual sense when $S$ is a smooth manifold (with or without boundary), or when $S$ is a convex set. The set of points $x$ for which $\partial_{P} S(x)$ is nontrivial (i.e., $\neq\{0\}$ ) can be "small", but is always dense in the boundary of $S$.

The (upper) Hamiltonian $H: \mathbb{R} \times \mathbb{R}^{n} \times \mathbb{R}^{n} \rightarrow \mathbb{R}$ is the function defined by

$$
H(t, x, p):=\max \{\langle p, v\rangle: v \in F(t, x)\} .
$$

Theorem 1. $\mathcal{R}$ is the unique closed subset $S$ of $\left[t_{0}, \infty\right) \times \mathbb{R}^{n}$ satisfying:

(i) $\theta+H(t, x, \zeta)=0 \quad \forall(\theta, \zeta) \in \partial_{P} S(t, x), \forall(t, x) \in\left(t_{0}, \infty\right) \times \mathbb{R}^{n}$,

(ii) $\lim _{T \downarrow 0} S_{T}=A$.

Remark 1. (a) Since $\partial_{P} S(t, x)$ is only defined when $(t, x)$ lies in $S$, the "proximal Hamilton-Jacobi equation" in (i) is in issue only at such points. Since $H(t, x, 0)=0$, it holds automatically at any point $(t, x) \in S$ for which $\partial_{P} S(t, x)$ is trivial.

(b) The initial condition (ii) is to be understood in the Hausdorff metric $\rho$; that is, for any $\varepsilon>0$ there exists $\delta>0$ such that

$$
T \in\left[t_{0}, t_{0}+\delta\right) \Rightarrow \rho\left(S_{T}, A\right)<\varepsilon .
$$

It follows in particular that $S_{t_{0}}=A$. It is equivalent to (ii) to require this last equality together with the uniform boundedness of $S_{T}$ for $T$ near $t_{0}$.

Proof of the Theorem. Let us verify first that $\mathcal{R}$ satisfies (i). Given any point $(\tau, \alpha)$ in $\mathcal{R}$, let $x$ be any trajectory on $[\tau, \infty)$ with $x(\tau)=\alpha$. We claim that $(t, x(t)) \in \mathcal{R}$ for all $t>\tau$. If $\tau=t_{0}$, then $\alpha \in A$ necessarily, and so $(t, x(t)) \in \mathcal{R}$ by the very definition of $\mathcal{R}$. If $\tau>t_{0}$, there is a trajectory $y$ on $\left[t_{0}, \tau\right]$ with $y\left(t_{0}\right) \in A, y(\tau)=\alpha$. But then " $y$ followed by $x "$ is a trajectory on $\left[t_{0}, \infty\right)$ beginning in $A$, whence $(t, x(t)) \in \mathcal{R} \quad \forall \sqcup>\tau$ as claimed. 
This argument implies that the set $\mathcal{R}$ is strongly invariant [1] [5] relative to the trajectories of $\widetilde{F}$, where $\widetilde{F}$ is the familiar augmented multifunction [5] [6]

$$
\widetilde{F}(t, x):=\{(1, v): v \in F(t, x)\} .
$$

This property is characterized [5] by the condition

$$
\theta+H(t, x, \zeta) \leq 0 \quad \forall(\theta, \zeta) \in \partial_{P} \mathcal{R}(\sqcup, \S), \quad \forall(\sqcup, \S) \in \mathcal{R} .
$$

We now proceed to observe another invariance property of $\mathcal{R}$. Let $(\tau, \alpha) \in \mathcal{R}, \tau>t_{0}$. Then there is at least one trajectory $x(\cdot)$ on $\left[t_{0}, \tau\right]$ such that $x(\tau)=\alpha, x\left(t_{0}\right) \in A$. Evidently, we have $(t, x(t)) \in \mathcal{R}$ for all $t \in\left[t_{0}, \tau\right]$. Thus $\mathcal{R}$ is weakly preinvariant for $\widetilde{F}[5]$ for $\tau>t_{0}$, which is a property characterized by the condition

$$
\theta+H(t, x, \zeta) \geq 0 \quad \forall(\theta, \zeta) \in \partial_{P} \mathcal{R}(\sqcup, \S), \quad \forall(\sqcup, \S) \in \mathcal{R}, \quad \sqcup>\sqcup,
$$

Combining (2) and (3) gives (i) of the theorem, for $S=\mathcal{R}$. That $\mathcal{R}$ satisfies property (ii) is an easy consequence of the fact that $F$ is bounded on compact sets; we omit the details.

Now let $S$ be another closed subset of $\left[t_{0}, \infty\right) \times \mathbb{R}^{n}$ satisfying (i) (ii). We first establish that $S \supseteq \mathcal{R}$. Let $(\tau, \alpha) \in \mathcal{R}$ for $\tau>t_{0}$. Then there is a trajectory $x(\cdot)$ on $\left[t_{0}, \tau\right]$ with $x\left(t_{0}\right)=: a \in A, x(\tau)=\alpha$. For any $\varepsilon>0$, we can find (by (ii)) $T \in\left[t_{0}, t_{0}+\varepsilon\right]$ and $a^{\prime} \in S_{T}$ such that $\left|a^{\prime}-a\right|<\varepsilon$. By continuous dependence of attainable sets, we can also suppose that there is a trajectory $x^{\prime}$ for $F$ on $[T, \tau]$ with $x^{\prime}(T)=a^{\prime}$ and $\left|x^{\prime}(\tau)-\alpha\right|<\varepsilon$. But $S$ is known to satisfy (2) (with $\mathcal{R}$ replaced by $S$, for $t>t_{0}$ ), which characterizes strong invariance relative to $\widetilde{F}$. Then, since $\left(T, a^{\prime}\right) \in S$, we have $\left(\tau, x^{\prime}(\tau)\right) \in S$ as well. Since $\varepsilon$ is arbitrary, it follows that $(\tau, \alpha) \in S$. This confirms the inclusion $S \supseteq \mathcal{R}$.

Now let $(\tau, \alpha)$ lie in $S, \tau>t_{0}$. Since $S$ satisfies (3) (with $\mathcal{R}$ replaced by $S$ ), $S$ is weakly preinvariant for $\widetilde{F}$ and for $t>t_{0}$. So for any $\varepsilon>0$, there is a trajectory $x_{\varepsilon}(\cdot)$ for $F$ on $\left[t_{0}+\varepsilon, \tau\right]$ such that $x_{\varepsilon}(\tau)=\alpha$, and such that $\left(t, x_{\varepsilon}(t)\right)$ lies in $S$ for all $t \in\left[t_{0}+\varepsilon, \tau\right]$. In particular, $x_{\varepsilon}\left(t_{0}+\varepsilon\right)$ lies in $S_{t_{0}+\varepsilon}$, which converges to $A$ as $\varepsilon \downarrow 0$. It follows from sequential compactness of trajectories [2, Theorem 3.1.7] that along some sequence of $\varepsilon_{i}$ decreasing to 0 , there is convergence of the corresponding $x_{\varepsilon_{i}}$ to a trajectory $x(\cdot)$ for $F$ on $\left[t_{0}, \tau\right]$ such that $x\left(t_{0}\right) \in A$ and $x(\tau)=\alpha$. Then $(\tau, \alpha) \in \mathcal{R}$. Thus $S \subseteq \mathcal{R}$.

Remark 2. If the Lipschitz condition (H3) is weakened to mere upper semicontinuity, it still follows that $\mathcal{R}$ is the maximal set satisfying (3) together with (ii). Thus $\mathcal{R}$ is a maximal "semisolution". This "comparison theorem" is another respect in which strong analogies exist with the various theories of generalized solutions of the HamiltonJacobi equation (proximal, minimax, viscosity: see [4] [5]). The evident analogy has a value function in the role of the set $\mathcal{R}$.

Remark 3. A connection between controllability and certain smooth approximations of the Hamilton-Jacobi equation has been obtained by Vinter [9]. The set $\mathcal{R}$ is of interest in connection with propogating fronts; we refer the reader to the interesting articles of Soravia [7] and Subbotin [8] for a functional approach to this issue.

\section{References}

[1] F. H. Clarke, Generalized gradients and applications. Trans. Amer. Math. Soc. 205 (1975), 247-262.

[2] __ Optimization and nonsmooth analysis, Wiley Interscience, New York, 1983; Classics Appl. Math., vol. 5, SIAM Publications, Philadelphia, 1990 (reprinted).

[3] _ Methods of dynamic and nonsmooth optimization, CBMS-NSF Regional Conf. Ser. in Appl. Math., vol. 57, SIAM Publications, Philadelphia, 1989.

[4] F. H. Clarke and Yu. S. Ledyaev, Mean value inequalities in Hilbert space, Trans. Amer. Math. Soc. 344 (1994) 307-324.

[5] F. H. Clarke, Yu. S. Ledyaev, R. J. Stern and P. R. Wolenski, Qualitative properties of trajectories of control systems: a survey, J. of Dynamical and Control Systems, (to appear).

[6] K. Deimling, Multivalued differential equations, De Gruyter, Berlin, 1992.

[7] P. Soravia, Generalized motion of a front propagating along its normal direction: a differential games approach, Nonlinear Anal. 22 (1994) 1247-1262. 
[8] A. I. Subbotin, Discontinuous solutions of a Dirichlet type boundary value problem for first order partial differential equations, Russian J. Numer. Anal. Math. Modelling 8 (1993) 145-164.

[9] R. B. Vinter, A characterization of the reachable set for nonlinear control systems, SIAM J. Control Optim. 18 1980, 599-610. 